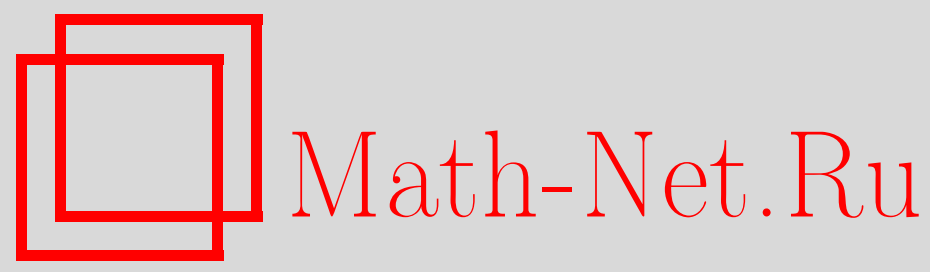

Д. В. Падучих, Влияние 2-окрестностей на строение графа, Матем. заметки, 1997, том 62, выпуск 6, 892-897

DOI: https://doi.org/10.4213/mzm1678

Использование Общероссийского математического портала Math-Net.Ru подразумевает, что вы прочитали и согласны с пользовательским соглашением http://www. mathnet.ru/rus/agreement

Параметры загрузки:

IP: 3.82 .47 .9

26 апреля 2023 г., 18:00:50

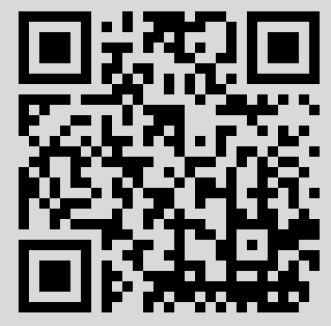




\title{
ВЛИЯНИЕ 2-ОКРЕСТНОСТЕЙ НА СТРОЕНИЕ ГРАФА
}

\author{
Д. В. Падучих
}

В работе описаны связные регулярные граффы диаметра большего 2, в которых 2-окрестности изоморфны граффу $\Delta$ диаметра 2 , а также регулярные графы диаметра 2 , в которых 2-окрестности изоморфны дистанционно регулярному графу $\Delta$ диаметра 3 . Доказано, что 2-локально графы Шрикханде не существуют.

Библиография: 2 названия.

В работе рассматриваются неориентированные графы без петель и кратных ребер. Под расстоянием $d(a, b)$ между вершинами $a, b$ графа Г понимается длина кратчайшей цепи, соединяющей $a \mathrm{c} b$. Назовем $i$-окрестностью вершины $a$ графа $\Gamma$ подграф $\Gamma_{i}(a)$, индуцированньй Г на множестве всех вершин графа $\Gamma$, которые находятся на расстоянии $i$ от вершины $a$. Если число вершин в $\Gamma_{i}(a)$ не зависит от выбора вершины $a \in \Gamma$, то оно обозначается через $k_{i}(\Gamma)$. Подграф $\Gamma_{1}(a)$ мы будем называть окрестностью вершины $а$ и обозначать через $[a]$. Через $a^{\perp}$ обозначим подграф $\{a\} \cup[a]$ (шар радиуса $1 \mathrm{c}$ центром $a$ ).

Пусть $\mathscr{F}$-некоторый класс графов. Граф Г назовем $i$-локально $\mathscr{F}$-графом, если $\Gamma_{i}(a)$ лежит в $\mathscr{F}$ для любой вершины $a$ графа $Г$. Если при этом класс $\mathscr{F}$ состоит из графов, изоморфных некоторому графу $\Delta$, то граф Г назовем $i$-локально $\Delta$-графом, при $i=1-$ локально $\Delta$-графом. Задача описания локально $\mathscr{F}$-графов является классической и решена для различных классов $\mathscr{F}$ (см., например, [1]). В данной работе исследуются 2-локально $\mathscr{F}$-графы.

Валентностью вершины назьвается число вершин в ее окрестности. Граф Г назьвается регулярным валентности $k$, если валентность любой вершины $a \in \Gamma$ равна $k$. Граф Г назьвается кореберно регулярным, если найдется натуральное число $\mu$ такое, что любые две несмежные вершины имеют точно $\mu$ общих соседей. Сильно регулярным графом с параметрами $(v, k, \lambda, \mu)$ назьвается $v$-вершинньй кореберно регулярный граф с соответствующим $\mu$, валентности $k$, в котором каждое ребро лежит в $\lambda$ треугольниках. Треугольным графом $T(m)$ называется граф с множеством неупорядоченных пар из множества $X$ в качествевершин, $|X|=m$, две пары $\{a, b\},\{c, d\}$ смежны тогда и только тогда, когда они имеют общий элемент. Будем говорить, что граф Г является прямой суммой своих подграфов $\Gamma_{i}$, если подграфы $\bar{\Gamma}_{i}$ являются связньгии компонентами графа $\bar{\Gamma}$.

Работа выполнена при финансовой поддержке Российского фонда фундаментальных исследований, грант № 96-01-00478. 
ЗАмЕчАниЕ. Пусть $\mathscr{F}$ - класс регулярных графов валентности $k$. Тогда связньй регулярньй 2-локально $\mathscr{F}$-граф Г диаметра 2 является кореберно регулярным и $\mu(\Gamma)=$ $k(\Gamma)-k$.

Если вершины $u, w$ находятся на расстоянии $i$ в $\Gamma$, то через $b_{i}(u, w)$ (соответственно

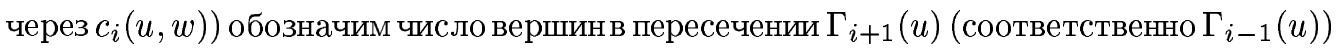
c $[w]$. Граф Г диаметра $d$ назьвается дистаниионно регулярным с массивом пересечений $\left\{b_{0}, b_{1}, \ldots, b_{d-1} ; c_{1}, \ldots, c_{d}\right\}$, если значения $b_{i}=b_{i}(u, w)$ и $c_{i}=c_{i}(u, w)$ не зависят от выбора вершин $u, w$ на расстоянии $i$ в $\Gamma$. Заметим, что для любого дистанционно регулярного графа с массивом пересечений $\left\{b_{i} ; c_{i}\right\}$ параметр $b_{0}$ - это валентность графа, $c_{1}=1$. Дистанщионно регулярный граф диаметра $d$ называется антиподальным, если отношение - находиться на расстоянии $d$ - является отношением эквивалентности на множестве вершин графа.

Сначала рассмотрим 2-локально $\Delta$-графы большого диаметра, в которых диаметр $\Delta$ равен 2. Графом Тейлора назьвается дистанционно регулярньй граф с массивом пересечений $\{k, \mu, 1 ; 1, \mu, k\}$. Регулярньй граф Г назовем редуиированным, если для любой вершины $a \in \Gamma$ подграф $\left\{x \in \Gamma \mid x^{\perp}=a^{\perp}\right\}$ состоит из единственной вершины $a$.

ПРЕДЛОЖЕНИЕ 1. Пусть $\Delta-$ регулярный граф диаметра 2. Тогда любой связный регулярный редуиированный 2-локально $\Delta$-граф Г диаметра большего 2 является графом Тейлора. В этом случае $\Delta$ - сильно регулярный граф $е k(\Delta)=2 \mu(\Delta)$. Обратно, для любого сильно регулярного графа $\Delta$ с $k(\Delta)=2 \mu(\Delta)$ найдется граф Тейлора $\Gamma$ такой, что $\Gamma_{2}(a) \simeq \Delta$ для любой вериины $a \in \Gamma$.

Диаметр Г ограничивается следующим утверждением.

ЛеммА. Пусть а - вериина графа $Г$ такая, что $\Gamma_{2}(a)$ - граф диаметра не больиего 3. Тогда а не лежит в геодезическом 4-пути вида bcade.

ДокАЗАТЕЛЬСтво. Если $b c a d e$ - геодезический 4-путь, то $b, e \in \Gamma_{2}(a)$, причем расстояние между $b, e$ в графе $\Gamma_{2}(a)$ не меньше 4 . Противоречие.

ДОКАЗАТЕЛЬСТВО ПРЕДЛОЖЕНИЯ 1. Пусть граф Г удовлетворяет условиям предложения $1, a$ - вершина из $\Gamma$ такая, что $\Gamma_{3}(a)$ непусто. Покажем сначала, что любая вершина $x \in \Gamma_{2}(a)$ смежна с любой вершиной $y \in \Gamma_{3}(a)$. В противном случае $\Gamma_{2}(a)$ содержит вершину $z$, находящуюся на расстоянии 2 от $y$. Противоречие с тем, что $\Gamma_{2}(z)$ содержит $a, y$ и расстояние в $Г$ между $a, y$ равно 3 .

Покажем теперь, что $\Gamma_{3}(a)$ является кликой (полным подграфом). В противном случае $\Gamma_{3}(a)$ содержит вершины $b, c$ c $d(b, c)=2$. Согласно лемме вершина $a \in \Gamma_{3}(b)$ должна быть смежна с $c$. Противоречие.

Итак, $x^{\perp}=y^{\perp}$ для любых вершин $x, y \in \Gamma_{3}(a)$. В силу редуцированности графа $\left|\Gamma_{3}(a)\right|=1$ и $[a]=\Gamma_{2}(b)$ для $b \in \Gamma_{3}(a)$. Ввиду регулярности графа $\Delta$ для любой вершины $c \in \Gamma_{2}(a)$ имеем $\mu(a, c)=|\Delta|-k(\Delta)-1$. Так как $\Gamma$ - регулярньй граф, то для любой вершины $x \in \Gamma$ подграф $\Gamma_{3}(x)$ непуст.

Таким образом, $\Gamma$ - дистанционно регулярный граф с массивом пересечений $\{|\Delta|$, $|\Delta|-k(\Delta)-1,1 ; 1,|\Delta|-k(\Delta)-1,|\Delta|\}$. По теореме Тейлора-Ливингстона [1, теорема 1.5.3] граф $\Delta$ является сильно регулярным с $k(\Delta)=2 \mu(\Delta)$.

Обратное утверждение следует из [1, теоремы $1.5 .2,1.5 .3]$.

Рассмотрим теперь графы диаметра 2 с 2-окрестностями больших диаметров. 
ПРЕДЛОЖЕНИЕ 2. Пусть Г - регулярный граф диаметра 2 и для любой вершинь $a \in \Gamma \quad \Gamma_{2}(a) \simeq \Delta$, где $\Delta-$ - егулярный связный граф. Тогда диаметр графа $\Delta$ не больие 3 .

ДокАЗАТЕЛЬСтво. Допустим, что диаметр $\Delta$ не меньше 4. Пусть $a \in \Gamma, b, c-$ вершины из $\Delta=\Gamma_{2}(a)$ такие, что $d_{\Delta}(b, c)=4$, и $b d e f c-$ кратчайший путь в $\Delta$. В силу замечания граф $\Gamma$ является кореберно регулярным, причем $[b] \cap[f]$ содержится в $[a]$, поэтому

$$
[a] \cap[f]=[a] \cap[b]=[b] \cap[c]=[d] \cap[c]=[a] \cap[d] .
$$

Противоречие с тем, что $[d] \cap[f]$ содержит еще вершину $e \in \Delta$.

ТеОрема 1. Пусть $\Delta$ - дистаниионно регулярный граф диаметра большего $2 c$ параметрами $(v, k, \lambda, \mu)$. Если $\Gamma$ - регулярный 2-локально $\Delta$-граф диаметра 2 , то выполняются следующие утверждения:

i) $\Delta$ - антиподальный дистаниионно регулярный граф диаметра 3 ;

ii) $\Gamma$ - прямая сумма графов $\Gamma_{i}, i=1, \ldots, n$, где $\Gamma_{i}-$ сильно регулярный әрафс параметрами

$v^{\prime}=v+1+\frac{k(k+1)}{k-\mu+1}, \quad k^{\prime}=\frac{k(k+1)}{k-\mu+1}, \quad \lambda^{\prime}=\frac{k^{2}+\mu-1}{k-\mu+1}-\frac{\mu v}{k+1}, \quad \mu^{\prime}=\frac{k \mu}{k-\mu+1}$.

Примеры графов, удовлетворяющих условиям теоремы 1 , дают графы, дополнительные к треугольным. В этих графах $\Delta$ является регулярньм двудольным графом с долями порядка $m$ и валентностью $m-1$. Граф Хоффмана-Синглетона (сильно регулярньй граф с параметрами $(50,7,0,1))$ также удовлетворяет условиям теоремы 1.

ДоКАЗАТЕЛЬСТвО ТЕОРЕмЫ 1. Пусть граф Г удовлетворяет условиям теоремы 1. Ввиду предложения $2 d(\Delta)=3$. Выберем вершину $a \in \Gamma$ и вершины $b, c$ на расстоянии 3 в графе $\Delta=\Gamma_{2}(a)$. Как и выше,

$$
[b] \cap[c]=[a] \cap[b]=[a] \cap[c]
$$

поэтому отношение - находиться на расстоянии 3 - является отношением эквивалентности на множестве вершин графа $\Delta$. Утверждение і) теоремы доказано.

Положим $r-1=k_{3}(\Delta)$ и покажем, что любая вершина $b \in[a]$ смежна в $\Delta$ либо с $r \mu$, либо со всеми вершинами. Действительно, пусть $c-$ несмежная с $b$ вершина из $\Delta$. Тогда $a, b \in \Gamma_{2}(c)$ и $[b] \cap[d]$ содержит $\mu$ вершин из $\Gamma_{2}(c) \cap \Delta$ для любого антипода $d$ к вершине $a$ в графе $\Gamma_{2}(c)$. Таким образом, вершина $b$ смежна с $(r-1) \mu$ вершинами из $\Gamma_{2}(c) \cap \Delta$ для любой несмежной с $b$ вершины $c \in \Delta$.

Так как для любой вершины $d \in \Delta$ такой, что $d_{\Delta}(c, d)=3$, имеем $[a] \cap[c]=[a] \cap[d]$, то $d_{\Gamma_{2}(c)}(a, d)=3$. Отсюда $d_{\Gamma_{2}(c)}(b, d)=2$, следовательно, вершины $b, d$ имеют $\mu$ общих соседей в $\Gamma_{2}(c)$. Значит, вершина $b$ смежна с $\mu^{\prime}-\mu$ вершинами из $[a] \cap[c]$, поэтому $b, c$ имеют точно $\mu$ общих соседей в $\Delta$.

Пусть $\Gamma^{\prime}$ - подграф, содержаший вершины из $[a]$, смежные с каждой вершиной из $\Delta$, $\Gamma_{0}=\Gamma-\Gamma^{\prime}$. Покажем, что граф $\Gamma$ является прямой суммой графов $\Gamma_{0}$ и $\Gamma^{\prime}$. Для этого достаточно показать, что любая вершина $b \in \Gamma_{0}(a)$ смежна с любой вершиной из $\Gamma^{\prime}$. По определению $\Gamma_{0}$ граф $\Delta$ содержит несмежную с $b$ вершину $c$. 
Рассмотрим $\Gamma_{2}(c)$. Очевидно, что каждая отличная от $b$ вершина $d$ из класса антиподов вершины $b$ в графе $\Gamma_{2}(c)$ лежит в $\Delta$, поэтому подграф $[c] \cap[d]=[b] \cap[c]$ содержит $\Gamma^{\prime}$.

Выше мы установили сильную регулярность графа $\Gamma_{0}$. Далее, граф $\Gamma^{\prime}$ удовлетворяет условиям теоремы, и по индукции граф $\Gamma^{\prime}$ является прямой суммой сильно регулярных графов. Нам осталось вычислить параметры графа $\Gamma$ в случае, когда $\Gamma$ - сильно регулярный граф.

Мы показали, что $b_{1}^{\prime}=b_{1}(\Gamma)=r \mu$. Подставив это выражение в прямоугольное соотношение $k^{\prime} b_{1}^{\prime}=k_{2}^{\prime} \mu^{\prime}$ для графа Г и учитьвая, что $k_{2}^{\prime}=r(k+1)$, получим $k^{\prime} \mu=(k+1) \mu^{\prime}$. Заменяя $k^{\prime}$ на $k+\mu^{\prime}$, имеем

$$
\mu^{\prime}=\frac{k \mu}{k-\mu+1} .
$$

Отсюда несложно вычислить и остальные параметры. Теорема 1 доказана.

Ситуация, в которой сам графф и его 2-окрестности имеют диаметр 2, представляется более сложной, чем рассмотренные выше. В некоторых задачах (см. [2]) по индукции для любой вершины $a \in \Gamma$ граф $\Gamma_{2}(a)$ оказывается графом Зейделя, т.е. сильно регулярным графом с наименьшим собственным значением -2. В связи с этим представляет интерес проблема описания связных регулярных графов, в которых 2-окрестности вершин являются графами Зейделя.

Мы рассмотрим один конкретньй случай этой проблемы.

ТЕОРема 2. Регулярные графы, в которых 2-окрестности вериин являются графами Шрикханде, не существуют.

ДокАЗАтЕльство. В графе на рис. 1 отождествим вершины $i^{+}, i^{-}(i=1,2, \ldots, 5)$. Затем отождествим вершины 1,5 и $j^{+}, j^{-}(j=6,7,8)$. Полученньй сильно регулярньй граф с параметрами $(16,6,2,2)$ назьвается графом Шрикханде.

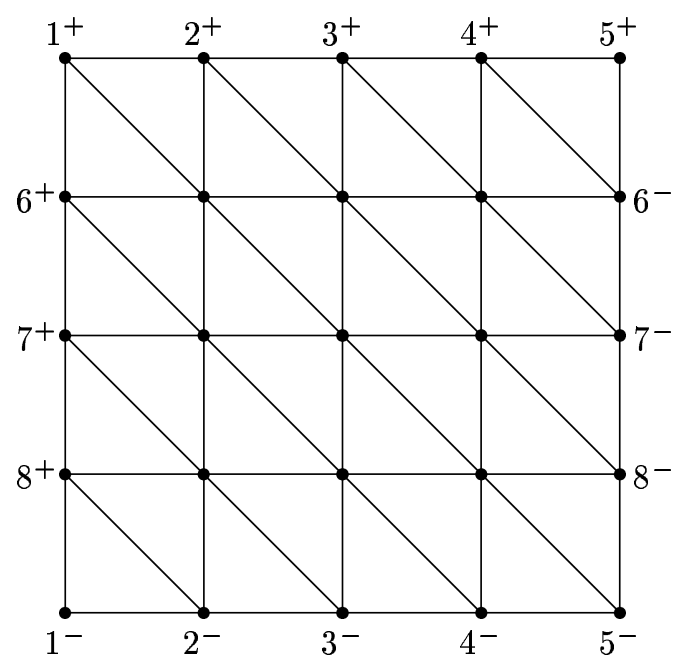

Рис. 1. Граф Шрикханде 


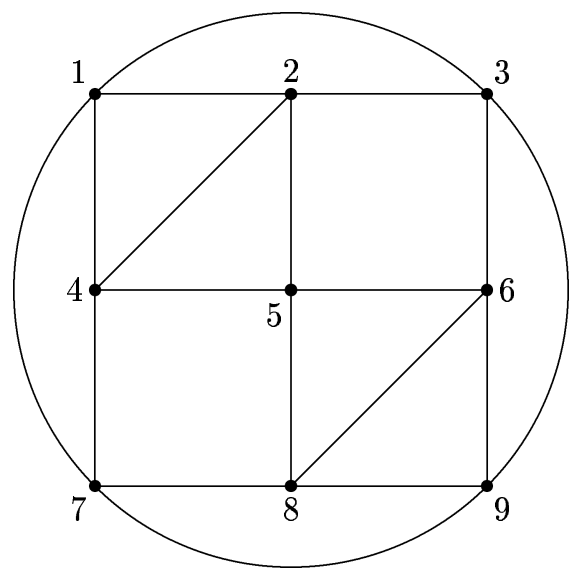

Рис. 2. Граф $\Delta_{0}$

Пусть граф Г удовлетворяет условиям теоремы 2. Из предложения 1 следует, что диаметр $\Gamma$ равен 2 , так как в графе Шрикханде $k \neq 2 \mu$. В силу замечания граф $\Gamma$ кореберно регулярен. Пусть $\Delta=\Gamma_{2}(a)$ для вершины $a \in \Gamma$. Тогда граф $\Delta_{0}=\Delta \cap \Gamma_{2}(b)$ для $b \in \Delta$ изображен на рис. 2 .

Пусть $c \in[a] \cap \Gamma_{2}(b)$. По строению графа $\Gamma_{2}(b)$ вершина $c$ смежна в $\Delta_{0}$ с одним из 2 -путей: $258,456,239,136,479,178$. Без ограничения общности можно считать, что вершина $c$ смежна с вершинами $2,5,8$. Далее, $\Delta(b)$ - шестиугольник, и точно три вершины, скажем $3,5,7$, смежны в нем с парами антиподальных вершин, а остальные вершины из $\Delta_{0}$ смежны с ребрами шестиугольника.

Пусть вершина 5 смежна с парой антиподальных вершин $5^{+}, 5^{-}$в шестиугольнике $\Delta(b)$. Очевидно, что $c \in \Gamma_{2}(4)$. Так как вершины 2,5 не лежат в $\Gamma_{2}(4)$, то $c$ смежна еще с двумя вершинами $d, e \in \Delta(b)$. Вершины $d, e, 8$ образуют 2 -путь в $\Gamma_{2}(4) \cap \Delta$, причем вершина 8 является крайней в этом 2-пути, так как она смежна с ребром ef графа $\Delta(b)$.
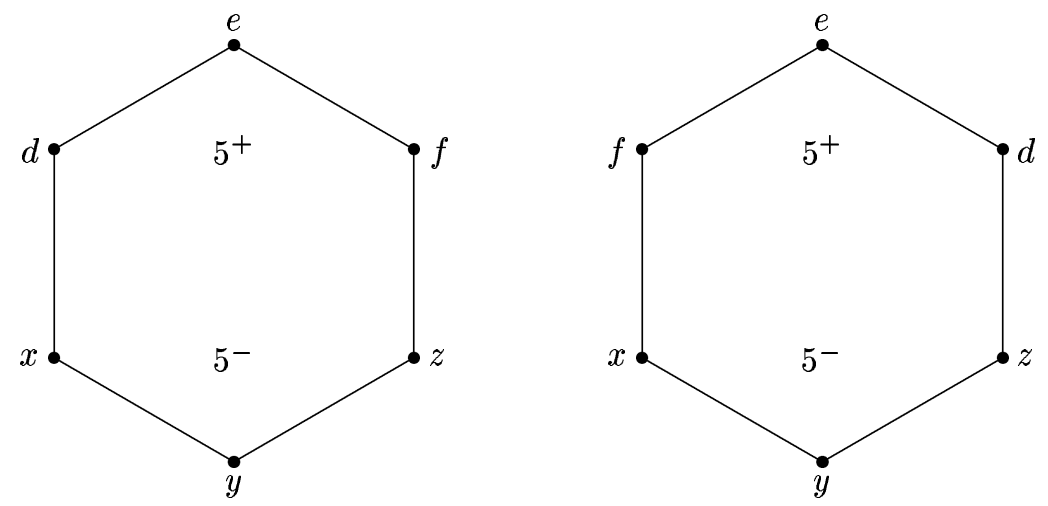

Рис. 3. Граф $\Delta(b)$

Напомним, что в графе Шрикханде $\mu=2$, поэтому вершины 2,8 имеют в $\Delta(b)$ единственного общего соседа. Аналогично, пары вершин 5,8 и 5,2 имеют по одному общему 
соседу в $\Delta(b)$. С другой стороны, вершины $5^{+}, 5^{-}$находятся на расстоянии 3 в $\Delta(b)$, следовательно, тройка вершин $2,5,8$ имеет в $\Delta(b)$ общего соседа. По симметрии то же самое верно для тройки вершин $4,5,6$.

Пусть вершина 4 смежна с ребром $x y$ графа $\Delta(b)$, а вершина 6 - с ребром $y z$ (см. рис. 3). Покажем, что вершина 2 смежна с ребром de.

В противном случае вершина 2 смежна с ребром $f w$ графа $\Delta(b)$ (см. рис. 4$)$. Рассмотрим подграф $\Gamma_{2}(6)$. Из рассуждений, проведенных для вершины 4 , следует, что вершина $c$ смежна с ребром в 2-пути $\Gamma_{2}(6) \cap \Delta(b)$. Противоречие с тем, что вершина $c$ смежна с $e$ и несмежна с $f$.

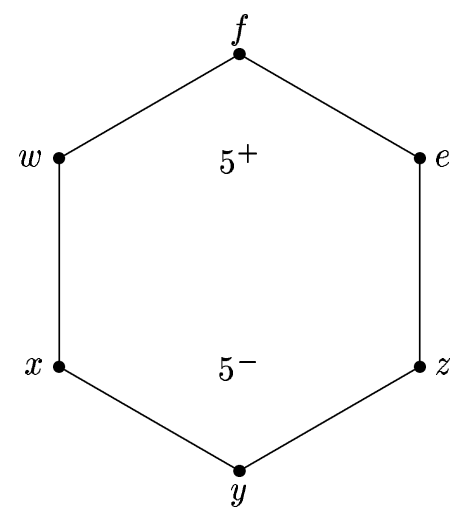

Рис. 4. Граф $\Delta(b)$

Таким образом, мы показали, что вершина 5 смежна с $e$. Но тогда одна из вершин 3,7 несмежна ни с $e$, ни с $d$. Пусть для определенности это будет вершина 3 . В этом случае вершина $c$ смежна с вершинами $5,8, e, d$ в $\Gamma_{2}(3) \cap \Delta(b)$. Противоречие. Теорема 2 доказана.

\section{СПИСОК ЦИТИРОВАННОЙ ЛИТЕРАТУРЫ}

[1] Brouwer A. E., Cohen A. M., Neumaier A. Distance-Regular Graphs. Berlin: Springer, 1989.

[2] Кабанов В. В., Махнев А. А. Кореберно регулярные графы без 3-лап // Матем. заметки. 1996. T. 60 . № 4. C. 495-503. 